\title{
STABILITY OF ATOMS AND MOLECULES IN AN ULTRARELATIVISTIC THOMAS-FERMI-WEIZSÄCKER MODEL
}

\author{
RAFAEL D. BENGURIA, MICHAEL LOSS AND HEINZ SIEDENTOP
}

\begin{abstract}
We consider the zero mass limit of a relativistic Thomas-FermiWeizsäcker model of atoms and molecules. We find bounds for the critical nuclear charges that insure stability.
\end{abstract}

\section{INTRODUCTION}

The zero mass limit of the relativistic Thomas-Fermi-Weizsäcker (henceforth ultrarelativistic TFW) energy functional for nuclei of charges $z_{i}>0$ (which need not be integral) located at $R_{i}, i=1, \ldots, K$ is defined by [3, 4]

$$
\xi(\rho)=a^{2} \int\left(\nabla \rho^{1 / 3}\right)^{2} d x+b^{2} \int \rho^{4 / 3} d x-\int V(x) \rho(x) d x+D(\rho, \rho),
$$

Here $\rho(x) \geq 0$ is the electron density,

$$
V(x)=\alpha \sum_{i=1}^{K} \frac{z_{i}}{\left|x-R_{i}\right|} .
$$

the electrostatic potential created by the nuclei,

$$
D(\rho, \rho)=\frac{\alpha}{2} \int \frac{\rho(x) \rho(y)}{|x-y|} d x d y,
$$

the electronic repulsion, and $\alpha=e^{2} / \hbar c \approx 1 / 137$ is the fine structure constant. In units in which $\hbar=c=1$, the constants $a^{2}$, and $b^{2}$ in (11) are given respectively by

$$
a^{2}=\frac{3}{8 \pi^{2}}\left(3 \pi^{2}\right)^{2 / 3} \lambda
$$

and

$$
b^{2}=\frac{3}{4}\left(3 \pi^{2}\right)^{1 / 3}
$$

In the non relativistic case, some emphasis has been placed on the question of an appropriate choice of the coefficient $\lambda$ of the gradient term connected to the kinetic energy. The non relativistic gradient correction $\lambda(\nabla \rho)^{2} / \rho$ was initially derived by Weizsäcker [14] with a value $\lambda=1$, whereas the systematic gradient expansion by Kirznits 7, 6] leads to $\lambda=1 / 9$. In the derivation of Tomishima and Yonei [13, $\lambda=1 / 5$. Lieb [8, 9] showed, that the Weizsäcker term introduces a $z^{2}$ correction

R.B. was supported by FONDECYT (Chile) projects \# 706-0200 and \# 106-0651 and CONICYT (Chile) PBCT Proyecto Anillo de Investigación en Ciencia y Tecnología ACT30/2006; M.L. was supported by NSF grant DMS-0600037; H.S. was supported by the Deutsche Forschungsgemeinschaft grant SI 348/13-1.

(C) 2007 by the authors. This paper may be reproduced, in its entirety, for non-commercial purposes. 
to the leading $z^{7 / 3}$ term of the non-relativistic ground state energy. Adapting the coefficient in front of the Weizsäcker term such that the correction agrees with the leading $z^{2}$ correction of the non-relativistic quantum mechanics, the Scott correction, lead Lieb to propose $\lambda=0.185$. In relativistic quantum mechanics, the leading energy correction remains unchanged whereas the Scott correction is smaller than in relativistic quantum mechanics [5], i.e., we cannot expect to have $\lambda$ the same value as in the TFW functional. However, we are not yet in a position to proceed with Lieb's strategy and to infer the coefficient of the gradient correction from this. In particular that would require showing that the massive equivalent of the function $\xi$ leaves indeed the leading energy contribution unchanged and the gradient term yields again a $z^{2}$ correction.

Let us first consider the atomic case, i.e., the case $K=1, z_{1}=z, R_{1}=0$. Because of simple scaling considerations, if we minimize the energy functional (1) over all functions $\rho$ for which each of the terms in (11) makes sense, the infimum of the energy functional is either zero or minus infinity. In the first case we say the atom is stable. Otherwise we say the atom is unstable. Our purpose here is to determine the range of values of the $z_{i}$ 's for which the atom or molecule is stable. The following result holds in the atomic case (i.e., for $K=1, z_{1}=z, R_{1}=0$ ) [1].

Theorem 1.1. Let

$$
\xi(\rho)=a^{2} \int\left(\nabla \rho^{1 / 3}\right)^{2} d x+b^{2} \int \rho^{4 / 3} d x-\int z \alpha \frac{\rho}{|x|} d x+D(\rho, \rho),
$$

with $D(\rho, \rho)$ given by (3). Then

$$
\inf \xi(\rho)=\left\{\begin{array}{lll}
-\infty & \text { for } & z>\frac{4 a b}{3 \alpha}+\frac{7 \pi a^{3}}{6 b^{3}} \\
0 & \text { for } & z<\frac{4 a b}{3 \alpha}
\end{array}\right.
$$

where the infimum is taken over all nonnegative functions $\rho(x)$, such that $\rho \in$ $L^{4 / 3}\left(\mathbb{R}^{3}\right), \nabla \rho^{1 / 3} \in L^{2}\left(\mathbb{R}^{3}\right)$, and $D(\rho, \rho)<\infty$.

\section{Remarks}

i) It follows from (17) that if $z<4 a b /(3 \alpha)$ the atom is stable, whereas if $z>$ $4 a b /(3 \alpha)+7 \pi a^{3} /\left(6 b^{3}\right)$ the atom is unstable. The exact critical value of $z\left(z_{c}\right.$ say) dividing the region of stability from the region of unstability is not known. However, it turns out that for the physical values of the constants the gap between the upper and lower bounds on $z_{c}$ is less than one, and therefore negligible (see the following remarks).

ii) For the physical values of $a$ and $b$ given by (4) and (5), the atom will be stable if $z<\sqrt{3 \lambda / 2} / \alpha \approx 167.8 \sqrt{\lambda}$. Thus, if $\lambda=1 / 9$ (i.e., the value used by Kirznits, [7, 6]) the atom is stable if $z<56$. If $\lambda=1 / 5$ (i.e., the value used by Tomishima and Yonei, [13]) the atom is stable if $z<75$. Finally, using the value of Lieb [8, 9], the atom is stable if $z<73$.

iii) As for the value of the gap, using the physical values of the constants, one gets $7 \pi a^{3} /\left(3 b^{6}\right)=(7 / 12 \pi) \sqrt{3 \lambda^{3} / 2}<0.021<1$ for all the values of $\lambda$ considered above. Thus, the gap is negligible from the physical point of view.

For the molecular case, i.e., when $K>1$, and $V$ is given by (2) the following result was proven in [1]. 
Theorem 1.2. Let

$$
\xi(\rho)=a^{2} \int\left(\nabla \rho^{1 / 3}\right)^{2} d x+b^{2} \int \rho^{4 / 3} d x-\int V \rho d x+D(\rho, \rho),
$$

with $V$ given by (2) and $D(\rho, \rho)$ given by (3). Then

$$
\inf \xi(\rho)=0 \quad \text { if } Z=\sum_{i=1}^{K} z_{i} \leq \frac{4 a b}{3 \alpha},
$$

where the infimum is taken over all nonnegative functions $\rho(x)$, such that $\rho \in$ $L^{4 / 3}\left(\mathbb{R}^{3}\right), \nabla \rho^{1 / 3} \in L^{2}\left(\mathbb{R}^{3}\right)$, and $D(\rho, \rho)<\infty$.

The above result is just a trivial extension of the atomic to the molecular case, and in some sense is the best possible when the interaction between the nuclei is not taken into account. In fact, if we neglect the nuclear interaction one can always think of the possibility of putting all the nuclear charges at the same point and reducing the molecular case to the atomic case, which explains the condition (9) on $Z \equiv \sum_{i=1}^{K} z_{i}$. The deficiencies of the above result are obvious. The goal of this paper is to have a result that yields stability for reasonable values of the nuclear charges. For that purpose the nucleus-nucleus interaction

$$
U \equiv \alpha \sum_{1 \leq i<j \leq K} \frac{z_{i} z_{j}}{\left|R_{i}-R_{j}\right|},
$$

plays a key role, because it prevents the possibility of putting the nuclear charges on top of each other.

Our main result is the following theorem for the molecular case.

Theorem 1.3. Let $\xi(\rho)$ be given by (8) for functions $\rho$ as in Theorem 1.2, with $V$ given by (2). Then, we have stability, i.e.,

$$
\inf \xi(\rho)+U \geq 0
$$

provided

$$
0 \leq z_{i} \leq \frac{4 a}{3 \alpha} b \sqrt{1-x}
$$

where $x \in(0,1)$ is the root of

$$
\frac{1-x}{x^{3}}=\frac{b^{4}}{a^{2}}\left(\frac{4}{3}\right)^{2} \frac{1}{2 \pi \alpha\left(4+9 \alpha^{4}\right)} .
$$

Remark For the physical values of $a$ and $b$ given by (4) and (5), and taken the physical value of the fine structure constant (i.e., $\alpha=1 / 137$ ) in (12), Theorem 1.3 says that the molecule will be stable if each $z_{i} \leq 55$, if $\lambda=1 / 9$ (i.e., the value used by Kirznits, [7, 6]). If $\lambda=1 / 5$ (i.e., the value used by Tomishima and Yonei, [13]) the molecule is stable if each $z_{i} \leq 74$. Finally, using the value of Lieb [8, 9], the molecule is stable if each $z_{i} \leq 71$. These bounds on each individual nuclear charge are almost the same as those embodied in the atomic case (i.e., the ones given in Theorem 1.1 above).

In the next section we give the proof of Theorem 1.3 . 


\section{IMPROVED RESULTS ON THE STABILITY OF MOLECULES}

In this section we prove Theorem 1.3 Our proof relies in a modified uncertainty principle (see Theorem 2.1 below) which is of independent interest. As we mentioned in the introduction, the nucleus-nucleus interaction plays a key role in the stability of molecules. As in [2] we may use the fact that the energy is separately concave in the nuclear charges and each charge $z_{i}$ varies between 0 and $z$. The minimum of a concave function is always on the boundary and hence the value od $z_{i}$ wants to be either 0 or $z$. If it is zero we have one nucleus less and if it is $z$ then we are in the case we are considering.

First we need some notation. We introduce the nearest neighbor, or Voronoi, cells [15] $\left\{\Gamma_{j}\right\}_{j=1}^{K}$ defined by

$$
\Gamma_{j}=\left\{x|| x-R_{j}|\leq| x-R_{k} \mid\right\} .
$$

The boundary of $\Gamma_{j}, \partial \Gamma_{j}$, consists of a finite number of planes. We also define the distance

$$
D_{j}=\operatorname{dist}\left(R_{j}, \partial \Gamma_{j}\right)=\frac{1}{2} \min \left\{\left|R_{k}-R_{j}\right| \mid j \neq k\right\} .
$$

One of the key ingredients we need in the sequel is an electrostatic inequality of Lieb and Yau [11, 12]. Define a function $\Phi$ on $\mathbb{R}^{3}$ with the aid of the Voronoi cells mentioned above. In the cell $\Gamma_{j}, \Phi$ equals the electrostatic potential generated by all the nuclei except for the nucleus situated in $\Gamma_{j}$ itself, i.e., for $x \in \Gamma_{j}$,

$$
\Phi(x) \equiv z \sum_{\substack{i=1 \\ i \neq j}}^{K}\left|x-R_{i}\right|^{-1} .
$$

If $\nu$ is any bounded Borel measure on $\mathbb{R}^{3}$ (not necessarily positive) then

$$
\frac{1}{2} \int_{\mathbb{R}^{3}} \int_{\mathbb{R}^{3}}|x-y|^{-1} d \nu(x) d \nu(y)-\int_{\mathbb{R}^{3}} \Phi(x) d \nu(x)+U \geq \frac{1}{8} z^{2} \sum_{j=1}^{K} D_{j}^{-1} .
$$

We will also need a localization result for the kinetic energy which will allow us to control the Coulomb potential near each nuclei. This localization result for the UTFW model is given by Theorem 2.1 below.

Theorem 2.1 (Modified uncertainty principle). For any smooth function $f$ on the closed ball $B_{R}$ of radius $R$ we have the estimate

$$
a^{2} \int_{B_{R}}|\nabla f(x)|^{2} d x+b^{2} \int_{B_{R}} f(x)^{4} d x \geq a b \int_{B_{R}}\left[\frac{4}{3|x|}-\frac{2}{R}\right] f(x)^{3} d x .
$$

Remark Notice that the factor $4 / 3$ is best possible and it agrees with the sharp value given in Theorem 1.1.

To prove this theorem, we need the following preliminary result.

Lemma 2.1. Let $u(r)$ be any smooth function with $u(R)=0$. Then the following uncertainty principle holds

$$
\left|\int_{B_{R}}\left(3 u(|x|)+u^{\prime}(|x|)|x|\right) f(x)^{3} d x\right| \leq 3\left(\int_{B_{R}}|\nabla f(x)|^{2} d x\right)^{1 / 2}\left(\int_{B_{R}} u(|x|)^{2}|x|^{2} f(x)^{4} d x\right)^{1 / 2} .
$$


There is equality if and only if

$$
f=\frac{1}{\lambda \int_{0}^{r}[s u(s)] d s+C}
$$

for some constants $C$ and $\lambda$.

Proof. Set

$$
g_{i}(x)=u(|x|) x_{i}
$$

where $u$ is a smooth function with $u(R)=0$ and note that

$$
\begin{gathered}
\int_{B_{R}}\left(3 u(|x|)+u^{\prime}(|x|)|x|\right) f(x)^{3} d x=\sum_{j} \int_{B_{R}} f(x)\left[\partial_{j} g_{j}(x)\right] f(x)^{2} d x \\
=\sum_{j} \int_{B_{R}} f(x) \partial_{j}\left(g_{j} f^{2}\right)(x) d x-2 \sum_{j} \int_{B_{R}} f(x)^{2} g_{j}(x) \partial_{j} f(x) d x .
\end{gathered}
$$

Integrating the first term by parts yields

$\sum_{j} \int_{B_{R}} f(x) \partial_{j}\left(g_{j} f^{2}\right)(x) d x=-\sum_{j} \int_{B_{R}} \partial_{j} f(x) g_{j}(x) f(x)^{2} d x+\int_{\partial B_{R}} f(x)^{3} u(|x|)|x| d S(x)$,

where the boundary term vanishes since $u(R)=0$. Thus,

$$
\int_{B_{R}}\left(3 u(|x|)+u^{\prime}(|x|)|x|\right) f(x)^{3} d x=-3 \sum_{j} \int_{B_{R}} f(x)^{2} g_{j}(x) \partial_{j} f(x) d x .
$$

Using Schwarz' inequality on the last term yields

$$
\left|\int_{B_{R}}\left(3 u(|x|)+u^{\prime}(|x|)|x|\right) f(x)^{3} d x\right| \leq 3\left(\int_{B_{R}}|\nabla f(x)|^{2} d x\right)^{1 / 2}\left(\int_{B_{R}} u(|x|)^{2}|x|^{2} f(x)^{4} d x\right)^{1 / 2} .
$$

Schwarz's inequality is an equality if and only if

$$
\partial_{j} f=-\lambda g_{j}(x) f(x)^{2},
$$

which can easily be integrated and yields the stated function.

Proof of Theorem 2.1. To prove the theorem, pick

$$
u(r)=\frac{1}{2}\left(\frac{1}{r}-\frac{1}{R}\right)
$$

in the lemma which leads to the inequality

$$
\int_{B_{R}}\left[\frac{1}{|x|}-\frac{3}{2 R}\right] f(x)^{3} d x \leq \frac{3}{2}\left(\int_{B_{R}}|\nabla f(x)|^{2} d x\right)^{1 / 2}\left(\int_{B_{R}}\left(1-\frac{|x|}{R}\right)^{2} f(x)^{4} d x\right)^{1 / 2}
$$

Next, applying the inequality between the arithmetic and geometric mean yields

$$
a^{2} \int_{B_{R}}|\nabla f(x)|^{2} d x+b^{2} \int_{B_{R}}\left(1-\frac{|x|}{R}\right)^{2} f(x)^{4} d x \geq a b \int_{B_{R}}\left[\frac{4}{3|x|}-\frac{2}{R}\right] f(x)^{3} d x,
$$

from which the theorem follows. 
In order to prove our main result, we consider the total energy, $\xi(\rho)+U$, and we split the $\int \rho^{4 / 3}$ term in two parts, i.e., $\left(b_{1}^{2}+b_{2}^{2}\right) \int \rho^{4 / 3}$. For later discussions, it is important to remark that the parameter $b_{2}$ can be chosen arbitrarily in the interval $(0, b)$. Then we use Theorem 2.1 with $f^{3}=\rho$ and $B_{R}=B_{j}$, the largest ball inscribed in the corresponding Voronoi cell $\Gamma_{j}$, to get

$$
\begin{aligned}
\xi(\rho)+U & \geq \quad b_{1}^{2} \int_{\mathbb{R}^{3}} \rho^{4 / 3} d x-\int_{\mathbb{R}^{3}} V(x) \rho(x) \\
& +\quad a b_{2} \sum_{j=1}^{K} \int_{B_{j}}\left(\frac{4}{3\left|x-R_{j}\right|}-\frac{2}{D_{j}}\right) \rho(x) d x+D(\rho, \rho)+U .
\end{aligned}
$$

In order to cancel the Coulomb singularity inside $B_{j}$ we choose the parameter

$$
b_{2}=\frac{3}{4} \frac{\alpha z}{a} .
$$

The restrictions on $b_{2}$ will give restrictions on $z$ to insure stability.

With the help of the Voronoi cells we now define,

$$
W(x) \equiv \Phi(x)+\frac{z}{\left|x-R_{j}\right|}
$$

if $x \in \Gamma_{j}$ and $\left|x-R_{j}\right| \geq D_{j}$, whereas,

$$
W(x) \equiv \Phi(x)+\frac{2 a b_{2}}{D_{j}},
$$

if $x \in \Gamma_{j}$ and $\left|x-R_{j}\right| \leq D_{j}$. Now, if we restrict to values of $z$ such that $z \leq$ $4 a b_{2} /(3 \alpha)$, we can finally write

$$
\xi(\rho)+U \geq \xi_{1}(\rho)+\xi_{2}(\rho)
$$

with

$$
\xi_{1}(\rho)=b_{1}^{2} \int_{\mathbb{R}^{3}} \rho^{4 / 3} d x-\alpha \int_{\mathbb{R}^{3}} W(x) \rho(x)+\alpha \int_{\mathbb{R}^{3}} \Phi \rho(x) d x,
$$

and

$$
\xi_{2}(\rho)=D(\rho, \rho)-\alpha \int_{\mathbb{R}^{3}} \Phi(x) \rho(x) d x+U
$$

Using the Lieb-Yau electrostatic inequality (16) we have

$$
\xi_{2}(\rho) \geq \alpha \frac{z^{2}}{8} \sum_{j=1}^{K} \frac{1}{D_{j}}
$$

On the other hand, it is simple to estimate $\xi_{1}(\rho)$ from below, since it is simple to solve the variational principle $\inf _{\rho} \xi_{1}(\rho)$. Thus we get,

$$
\xi_{1}(\rho) \geq-\frac{1}{4} \alpha^{4}\left(\frac{3}{4 b_{1}^{2}}\right)^{3} \int_{\mathbb{R}^{3}}(W-\Phi)_{+}^{4} d x .
$$

From (21), (24), and (25) we get

$$
\xi(\rho)+U \geq-\frac{1}{4} \alpha^{4}\left(\frac{3}{4 b_{1}^{2}}\right)^{3} \int_{\mathbb{R}^{3}}(W-\Phi)_{+}^{4} d x+\alpha \frac{z^{2}}{8} \sum_{j=1}^{K} \frac{1}{D_{j}} .
$$

Now, using (20) we compute,

$$
\int_{B_{j}}(W-\Phi)_{+}^{4} d x=\left(\frac{2 a b_{2}}{D_{j}}\right)^{4} \frac{4}{3} \pi D_{j}^{3}=\frac{64 \pi a^{4} b_{2}^{4}}{3 D_{j}} .
$$


Since any Voronoi cell is contained in a half space, one calculates as in [10] that

$$
\int_{\Gamma_{j} \backslash B_{j}}(W-\Phi)_{+}^{4} d x=\int_{\Gamma_{j} \backslash B_{j}}\left(\frac{z}{\left|x-R_{j}\right|}\right)^{4} d x \leq \frac{3 \pi z^{4}}{D_{j}} .
$$

Finally, using the estimates (27) and (28) in (26), we get

$$
\xi(\rho)+U \geq \frac{\alpha}{8} M \sum_{j=1}^{K} \frac{1}{D_{j}},
$$

where

$$
M=-\frac{1}{4} \alpha^{4}\left(\frac{3}{4 b_{1}^{2}}\right)^{3}\left(3 \pi z^{4}+\frac{64 \pi a^{4} b_{2}^{4}}{3}\right)+\frac{\alpha z^{2}}{8} .
$$

If $M>0$ then $\xi(\rho)+U>0$ and the molecule is stable. Using the fact that $b_{2}^{2}=b^{2}-b_{1}^{2}$ together with (18) the condition $M \geq 0$ can be written solely in term of $b_{1}$ as

$$
a^{2} \frac{b^{2}-b_{1}^{2}}{b_{1}^{6}} \leq\left(\frac{4}{3}\right)^{2} \frac{1}{2 \pi\left(4 \alpha+9 \alpha^{5}\right)} .
$$

Finally, in order to allow for the largest possible value of $z$ (equivalently the largest possible value of $\left.b_{2}\right), b_{1}$ must be chosen so that we have equality in (31). Since the left side of (31) is decreasing as a function of $b_{1}$ in the allowed interval $(0, b)$, we conclude the proof of our Theorem 1.3 .

\section{Remarks}

i) Note that we do not need a bound on the fine structure constant to ensure stability in this model. This is due to the absence of the exchange term.

ii) If we set $\alpha=\frac{1}{137}$ we find stability up to $z=71$, when the parameter $\lambda=0.185$, up to $z=74$ when $\lambda=0.2$ and $z=55$ when $\lambda=1 / 9$.

\section{REFERENCES}

[1] R. D. Benguria and S. Pérez-Oyarzún, The ultrarelativistic Thomas-Fermi-von Weizsäcker model, J. Phys A: Math. \& Gen. 35 (2002), 3409-3414.

[2] E. H. Lieb and I. Daubechies, One electron relativistic molecules with Coulomb interaction, Commun. Math. Phys. 90 (1983), 497-510.

[3] E. Engel and R. M. Dreizler, Field-theoretical Approach to a Relativistic Thomas-FermiWeizsäcker Model, Phys. Rev. A 35 (1987), 3607-3618.

[4] E. Engel and R. M. Dreizler, Solution of the relativistic Thomas-Fermi-Dirac-Weizsäcker Model for the Case of Neutral Atoms and Positive Ions, Phys. Rev. A 38 (1988), 3909-3917.

[5] R. L. Frank, H. Siedentop, and S. Warzel, The Ground State Energy of Heavy Atoms: Relativistic Lowering of the Leading Energy Correction, Commun. Math. Phys. (in press).

[6] C. H. Hodges, Quantum Corrections to the Thomas-Fermi Approximation -The Kirzhnits Method, Can J. Phys. 51 (1973), 1428-1437.

[7] D. A. Kirznits, Field Theoretical Methods in Many Body Systems, Pergamon, NY, 1967.

[8] E. H. Lieb, Thomas-Fermi and Related Theories of Atoms and Molecules, Reviews in Modern Physics 53 (1981), 603-641.

[9] E. H. Lieb, Analisis of the Thomas-Fermi-von Weizsäcker Equation for an Infinite Atom without Electron Repulsion, Commun. Math. Phys. 85 (1982), 15-25.

[10] E. H. Lieb, M. Loss and H. Siedentop, Stability of Relativistic Matter via Thomas-Fermi Theory, Helv. Phys. Acta 69 (1996), 974-984.

[11] E. H. Lieb and H. Yau, Many-Body Stability Implies a Bound on the Fine-Structure Constant, Phys. Rev. Lett. 61 (1988), 1695-1697. 
[12] E. H. Lieb and H. Yau, The Stability and Instability of Relativistic Matter, Commun. Math. Phys. 118 (1988), 177-213.

[13] Y. Tomishima and K. Yonei, Solution of the Thomas-Fermi-Dirac Equation with a Modified Weizsäcker Correction, J. Phys. Soc. Jpn. 21 (1966), 142-153.

[14] C. F. von Weizsäcker, Zur Theorie de Kernmassen, Z. Physik 96 (1935), 431-458.

[15] G. Voronoi (1907), Nouvelles applications des paramètres continus à la théorie des formes quadratiques, Journal für die Reine und Angewandte Mathematik 133 (1907), 97-178.

E-mail address: rbenguri@fis.puc.cl, loss@math.gatech.edu, h.s@lmu.de

Department of Physics, Pontificia Universidad Católica de Chile Casilla 306, Correo 22 Santiago, Chile

School of Mathematics, Georgia Institute of Technology, Atlanta, Ga 30332

Mathematisches Institut, Ludwig-Maximilians-Universität München, Theresienstrasse 39, 80333 München, Germany 